\title{
Itinéraires
}

Itinéraires Littérature, textes, cultures

2010-2 | 2010

\section{Les blogs}

\section{Bibliographie}

\section{(2) OpenEdition \\ 1 Journals}

Édition électronique

URL : http://journals.openedition.org/itineraires/2093

DOI : 10.4000/itineraires.2093

ISSN : 2427-920X

Éditeur

Pléiade

\section{Édition imprimée}

Date de publication : 1 juillet 2010

Pagination : 193-197

ISBN : 978-2-296-12012-9

ISSN : $2100-1340$

Référence électronique

«Bibliographie », Itinéraires [En ligne], 2010-2 | 2010, mis en ligne le 01 juillet 2007, consulté le 15 septembre 2020. URL : http://journals.openedition.org/itineraires/2093

\section{(c) (1) () $\Theta$}

Itinéraires est mis à disposition selon les termes de la licence Creative Commons Attribution - Pas d'Utilisation Commerciale - Pas de Modification 4.0 International. 


\section{Bibliographie}

Cette bibliographie consacrée aux blogs est complétée par un ensemble de références concernant les écrits d'écran et la notion de genre, dans une version électronique consultable en ligne sur le site du CENEL : http://www. univ-paris 13.fr/cenel/articles.htm.

\section{Ouvrages}

Assouline, Pierre, Brèves de blog, Paris, Les Arènes, 2008.

Cauquelin, Anne, L'Exposition de soi. Du journal intime aux Webcams, Paris, Eshel, 2003.

Desavoye, Benoît, Ducamp, Christophe, Mazenod, Xavier de et Moisant, Xavier, Les Blogs. Nouveau média pour tous, Paris, M2 éditions, 2005.

Ertzscheid, Olivier, Créer, trouver et exploiter les blogs, Paris, ADBS éditions, 2008. Fiévet, Cyril et Turretini, Emily, Blog story, Paris, Eyrolles, 2004.

Lejeune, Philippe, « Cher écran ... », journal personnel, ordinateur, internet, Paris, Seuil, 2000. Suite chronologique de l'exploration : <http://www.autopacte.org/un_an_apr\%E8s. $\mathrm{html}>$. Bibliographie : <http://www.autopacte.org/Etudes_recentes.html $>$.

Le Meur, Loïc et Beauvais, Laurence, Blogs \& podcasts, Paris, Dunod, 2007.

Lovink, Geert, Zero Comments : Blogging and Critical Internet Culture, New York, Routledge, 2008.

Michel, Serge (L'Hebdo), Bondy blog. Des journalistes suisses dans le 9.3, Paris, Seuil, 2006.

Walker Rettberg, Jill, Blogging, Cambridge, Polity Press, 2008. Voir aussi : <jill-txt.net>.

\section{Numéro de revue}

«Les blogs », présentation de Dominique Cardon, Valérie Jeanne-Perrier, Florence Le Cam et Nicolas Pélissier, Réseaux, Paris, vol. 24, n 138, 2006.

\section{Articles et communications}

Allard, Laurence et Vandenberghe, Frédéric, « Express yourself! Les pages perso entre légitimation technopolitique de l'individualisme expressif et authenticité réflexive peer to peer », Réseaux, « Les nouvelles formes de la consécration culturelle », n 117, 2003. 
Allard, Laurence, « Termitières numériques : les blogs comme technologie agrégative de soi », Multitudes, "Postmédia, réseaux, mise en commun », $\mathrm{n}^{\circ}$ 21, 2005.

Ammam, Rudolf, « Jorn Barger, the NewsPage Network, and the Emergence of the weblog Community ", dans HT'09: Proceedings of the twentieth ACM conference on hypertext and hypermedia, 2009, <http://tawawa.org/ark/p/jorn-barger-community.html>.

Arocamora, Agnès et Bartlett, Djurdja, « Blogs de mode : les nouveaux espaces du discours de mode », Sociétés, vol. 2, n 104, 2009, p. 105-114.

Barrett, Cameron, « Anatomy of a weblog », dans John Rodzvilla (éd.), We've got blog: How weblogs are changing our culture, Cambridge, Mass., Perseus Publishing, 2002. Bary, Cécile de, « Les blogs. Un effet littéraire ? », RiLUnE, n 5, juillet 2006.

Bellanger, Pierre, « Des radios libres aux skyblogs. Entretien », Le Débat, « Penser les médias II », n 139, mars-avril 2006.

Blood, Rebecca, « Weblogs : A History and Perspective », 2000 (mise en ligne), $<$ http://www.rebeccablood.net/essays/weblog_history.html $>$.

Boyd, Danah, « Broken Métaphores as Liminal Practice », 2004, <www.danah. org/papers/BrokenMetaphors.pdf>.

Broudoux, Évelyne, « Je blogue, tu blogues, nous bloguons - du carnet individuel à l'écriture collective », Un point d'actu, supplément en ligne à la revue Les dossiers de l'ingénierie éducative, $\mathrm{n}^{\circ}$ 45, « Publier en ligne aujourd'hui », décembre 2003.

—, «Autorités énonciatives et espaces de publication et de référencement », janvier 2006, sur le site Urfist (actualité des sciences de l'information), <http://www. urfistinfo.blogs.com>.

Candel, Étienne et Jeanne-Perrier, Valérie, « Blogs, vous avez dit blogs ? », dossier réalisé pour le projet du site « Mediata », Celsa, 2006, <http://www.celsa.fr>.

Chapelain, Brigitte, «Les blogs d'écrivains : un nouvel enjeu pour les littératures francophones », dans Larbi Chouikha, Vincent Meyer et Wahid Gdoura (dir.), Interagir et transmettre, informer et communiquer : quelles valeurs, quelles valorisations?, actes du colloque international des sciences de l'information et de la communication, Tunis, ISD, IPSI, SFSIC, 2008.

Delaunay-Téterel, Hélène, «Sociabilité juvénile et construction de l'identité. L'exemple des blogs adolescents », Informations sociales, ${ }^{\circ}$ 145, 2008.

Deseilligny, Oriane, «L'écriture de journaux intimes sur Internet : mise en forme du moi ou création d'une image de soi ? », dans Hypertextes et Hypermédias : créer $d u$ sens à l'ère numérique, H2PTM'03, Paris, Hermès science, septembre 2003.

—, « Du journal intime au blog », Communication \& Langages, $\mathrm{n}^{\circ} 150$, mars 2008.

Dibbell, Julian, « Portrait of the Blogger as A Young Man », dans John Rodzvilla (éd.), We've got blog : How weblogs are changing our culture, Cambridge, Mass., Perseus Publishing, 2002.

Ertzscheid, Olivier, « Weblogs : un nouveau paradigme pour les systèmes d'information et la diffusion de connaissances ? Applications et cas d'usage en contexte de veille et d'intelligence économique », dans Organisation des connaissances dans les systèmes d'informations orientés utilisation : contexte de veille et d'intelligence économique, colloque Isko-France 2005, 28-29 avril 2005. 
Fluckiger, Cédric, « La sociabilité juvénile instrumentée. L'appropriation des blogs dans un groupe de collégiens ", Réseaux, n 138, 2006.

Fraissard, Guillaume et Zilbertin, Olivier, « Quand les journalistes font blog à part. Dévoiler les coulisses, relater les petites phrases... Internet permet-il de s'affranchir des règles de l'art? ", Le Monde, 8-9/10/2006.

Gary, Nicolas, « Le blog est dépassé, aujourd'hui on écrit des romans sur Twitter », complément Web à Books, $\mathrm{n}^{\circ}$ 7, juillet-août 2009, <http://www.booksmag.fr/magazine/c/nicolas-gary-le-blog-est-depasse-aujourd-hui-on-ecrit-des-romans-sur-twitter-1.html > .

Herring, Susan, Scheidt, Lois, Bonus Sabrina et Wright, Elijah, « Weblogs as a Bridging Genre », dans Information, Technology 1 People, $\mathrm{n}^{\circ} 18$ (2), 2005.

Jacobs, Joanne, « Communications Over Exposure : The Rise of Blogs as a Product of Cybervoyeurism », ANZCA 03 Conference, Brisbane, Australia, 2003, <http://www. bgsb.qut.edu.au/conferences/ANZCA03/Proceedings/papers/jjacobs_full.pdf $>$.

Jeanne-Perrier, Valérie, « Des outils d'écriture aux pouvoirs exorbitants? », Réseaux, $\mathrm{n}^{\circ} 137,2006 / 3$.

Klein, Annabelle, « Les homepages, nouvelles écritures de soi, nouvelles lectures de l'autre », Spirale, n 28, Lille, 2001.

Laudouar, Janique, « Désir de s'exposer, désir d'écrire », Un point d'actu, supplément en ligne à la revue Les dossiers de l'ingénierie éducative, $\mathrm{n}^{\circ} 45$, « Publier en ligne aujourd'hui », Cndp, décembre 2003, <http://www.cndp.fr/archivage/valid/54656/54656-7748-7732.pdf>.

Le Cam, Florence, «États-Unis : les weblogs d'actualité ravivent la question de l'identité journalistique ", Réseaux, n 138, 2006.

—, « Histoires et filiations du terme “weblog” (1992-2003). Perspectives pour penser l'histoire de certaines pratiques sociales sur le Web », communication du 12 juin 2008, mis en ligne le 15 avril 2010, <http://w3.u-grenoble3.fr/les_enjeux//2010/ LeCam/index.html>.

Lialina, Olia, « Vernacular web », conférence Decade of web Design, Amsterdam, 21-22 janvier 2005, <http://art.teleportacia.org/observation/vernacular/>.

Lialina, Olia, « Vernacular web 2 or Rich User Experience for the Poor », conférence New Network Theory, 28-30 juin 2007, <http://contemporary-home-computing. org/vernacular-web-2/>.

Madre, Frédéric, « Blog : un chien parmi les chiens, contraintes », Formules, $\mathrm{n}^{\circ} 10$, juin 2006.

Mortensen, Torill et Walker, Jill, «Blogging Thoughts : Personal Publication as Online Research Tool », dans Andrew Morrison (éd.), Researching ICTs in Context, InterMedia Report, mars 2002, Oslo, 2002.

Pagès, Jean-Luc, « Blog littéraire/blog personnel », notice en cours d'élaboration, prochainement en ligne sur le site du Dictionnaire international des termes littéraires (DITL), <www.flsh.unilim.fr/ditl/>.

Ramocki, Martin, « Surfing Clubs : organized notes and comments », conférence NSCAD : "Obsolescence and the Culture of Human Invention », Halifax, 28 mai 2008, <http://ramocki.net/surfing-clubs.html>. 
Thompson, Gary, « Visual Factors in Constructing Authenticity in weblogs », Toronto (Canada), 2003, <http://aoir.org/members/papers42/Thompson_visauthdraft.htm>. Trédan, Olivier, Le phénomène des blogs, rapport d'étude, Marsouin, mis en ligne le 3 juin 2005, 126 p., <http://expert.infini.fr/Le-phenomene-des-blogs>.

Walker, Jill, « Mirrors and Shadows : The Digital Aestheticisation of Oneself », dans The Proceedings of Digital Arts and Culture, IT University, Copenhague, décembre 2005, <https://bora.uib.no/bitstream/1956/1136/1/mirrorsandshadows-final.pdf>.

\section{Communications et colloques non encore publiés :}

« Hypertextes, mémoire, fiction », colloque à l'université de Montréal, novembre 2003, avec notamment une intervention de Broudoux, Évelyne, «Autoritativité, support informatique, mémoire ».

« Les blogs : récits de soi, écritures collectives sur le monde ou journalisme ? », table ronde organisée lors du colloque Les écritures d'écran : histoire, pratiques et espaces sur le Web, Aix-en-Provence, Maison méditerranéenne des Sciences de l'Homme, 18 et 19 mai 2005. Parmi les interventions : Deseilligny, Oriane, « De la destination des journaux intimes en ligne »; Guillaud, Hubert, « Tentative de typologie des formes éditoriales des blogs »; Rebillard, Franck, « Des blogs au journalisme ».

Lejeune, Philippe et Massip, Bernard, « La littérature personnelle sur Internet », conférence à l'ENS de Lyon, 27 janvier 2006.

« Les sites internet : description et exploitation en langue », journée d'étude à l'ENS LSH de Lyon, 16 mars 2006. Parmi les interventions : Richer, Jean-Jacques, « Essai de définition du blog comme genre de discours »; Soubrié, Thierry, « Caractéristiques sémiopragmatiques des blogs et pistes d'exploitation pédagogique ».

Soufron, Jean-Baptiste, « Les blogs, leur rôle et leurs limites », journée d'étude Les données personnelles et les nouveaux outils d'échange organisée par le CREIS, Paris, 9 juin 2006.

Soubrié, Thierry, «Le blog : fonction cognitive et réhabilitation de la figure de l'auteur », colloque international Jocair'2006, université d'Amiens, juillet 2006.

\section{Billets}

Bon, François, « Tumulte », <http://www.tierslivre.net/livres/tumulte>.

—, « 130 blogs littérature et Internet », <http://www.netvibes.com/tierslivre\#tiers_livre>.

Crouzet, Thierry, « Vers un web sans site web », Le peuple des connecteurs, 17 août 2009, <http://blog.tcrouzet.com/2009/08/17/vers-un-web-sans-site-web>.

Druaux, Christophe, "Cartographie subjective de la blogarchie francophone », $<$ http://www.ouinon.net/index.php?2007/09/24/215-cartograhie-blogosphere-francophone>.

Dussidour, Dominique, «"Les livres nous accompagnent même dans nos chutes" », 2 décembre 2006, <http://remue.net/spip.php?article1987>.

Genin, Christine, parcours guidé sur le blog des lecteurs de la BnF : blogs d'écrivains, $<$ http://blog.bnf.fr/lecteurs/index.php/2009/03/31/decouvrir-des-blogs-d-ecrivains/> ; journaux personnels, $<$ http://blog.bnf.fr/lecteurs/index.php/2009/04/06/du-journalintime-au-blog/>. 
Giffard, Alain, « Sur le blog », 13 mai 2005, <http://alaingiffard.blogs.com/culture/2005/05/sur_le_blog_1_.html>.

—, « Skyblog, la grande "secte” molle ? », 21 mars 2009, <http://alaingiffard.blogs. com/culture/2009/03/skyblog-la-grande-secte-molle.html $>$.

Girardeau, Astrid, «C'est quoi, un blog ? », Écrans, 19 décembre 2007, <http://www. ecrans.fr/C-est-quoi-un-blog,2836.html>.

Gunthert, André, « Why blog ? », Actualités de la Recherche en histoire visuelle, 15 septembre 2008, <http://www.arhv.lhivic.org/index.php/2008/09/15/807-why-blog>.

Herring, Susan, Scheidt, Lois, Bonus Sabrina et Wright, Elijah, « Bridging the Gap : A Genre Analysis of weblogs », 2004, <http://www.blogninja.com/index.php>.

Ito, Joichi, «weblogs and Emergent Democracy », 10 août 2004, <http://joi.ito. com/static/emergentdemocracy.html $>$.

Maïsetti, Arnaud, « Internet, de la condition du blogueur », 23 février 2009, <http:// arnaudmaisetti.net/spip/spip.php?article10>.

Moss, Ceci et Boling, John Michael, « General web Content : tumblr blogs », Rizhome, 26 janvier 2009, <http://rhizome.org/editorial/2386>.

Pagé, Martine, « J'ai finalement créé mon propre blog », 27 février 2002, <http:// www/largeur.com/expArt.asp?artID=109>.

Pisani, Francis, « Blogalaxie/9 - 133 millions de blogueurs, 1,5 million d'actifs », Transnets, 24 septembre 2008, <http://pisani.blog.lemonde.fr/2008/09/24/blogalaxie9-133-millions-de-blogueurs-15-million-dactifs/>.

Sifry, David, « State of the blogosphere », Technorati, 23 septembre 2008, <http:// www.technorati.com/blogging/state-of-the-blogosphere/>.

Spivak, Nova, « Welcome to the Stream », Twine, 8 mai 2009, <http://www.twine. com/item/1281ryv9z-46/is-the-stream-is-the-next-new-metaphor $>$.

Valclair, « Blogueurs du temps court, blogueurs du temps profond », Les échos de Valclair, 11 juin 2009, <http://valclair.canalblog.com/archives/2009/06/11/14045679. html>.

Véronis, Jean, « Web : Google, Blogger et le splog », Technologies du langage, 9 septembre 2005, <http://aixtal.blogspot.com/2005/09/web-google-blogger-et-lesplog.html>.

\section{Du côté des blogs}

Pointblog, <www.pointblog.com> : définitions, lexique, information.

Médiatic blogspot, <http://mediatic.blogspot.com>.

L'Écume des blogues, <http://lecumedesblogues.blogspot.com> : revue de la blogosphère.

« Weblog webliography », <http://kairosnews.org/blogbib>.

« Master list of blog articles », <http://www.ourboldhero.com/research/masterlist. html $>$ (dernière mise à jour le 20 décembre 2006).

« An Annotated Bibliography on weblogs and Blogging », BlogBib, <http://blogbib.blogspot.com/>. 